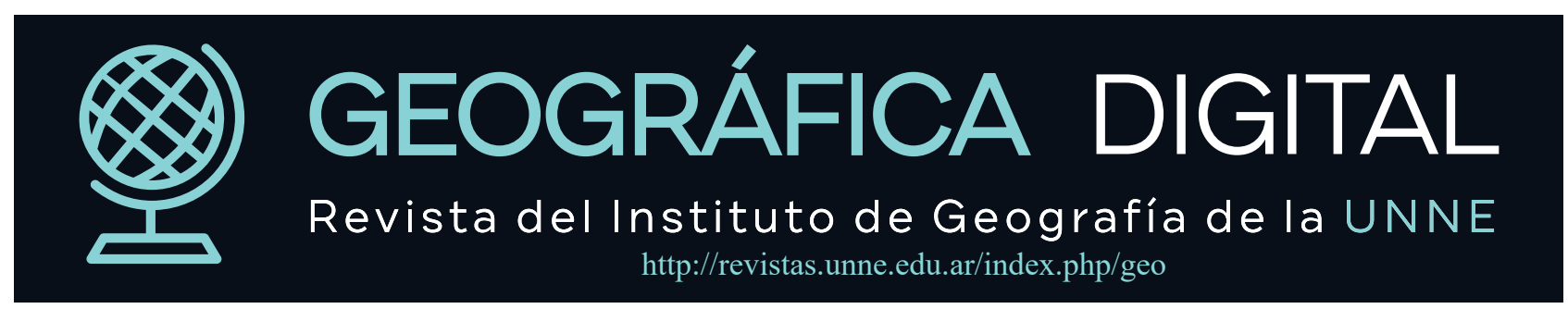

Revista Geográfica Digital, 2020, Vol. 17, N³3, 2-16 pp., E-ISSN: 1668-5180: DOI: http://dx.doi.org/10.30972/geo.17334097

\title{
Expansión y densificación urbana del Gran Buenos Aires (2012-2019) a partir de imágenes satelitales nocturnas
}

\author{
Urban Expansion and densification of Gran Buenos Aires (2012-2019) from \\ nocturnal satellite images
}

\begin{abstract}
Montes Galbán, Eloy
Instituto de Investigaciones Geográficas. Universidad Nacional de Luján / Consejo Nacional de Investigaciones Científicas y Técnicas (CONICET). emontesgalban@gmail.com
\end{abstract}

Palabras Claves:

Expansión urbana

Gran Buenos Aires

Imágenes satelitales nocturnas Suomi NPP VIIRS

luminosidad urbana

\section{R E S U M E N}

El objetivo del presente trabajo consistió en analizar la expansión y densificación urbana del Gran Buenos Aires (GBA) para el periodo 2012 - 2019, a partir de imágenes satelitales nocturnas de la Tierra. Se realizó una primera aproximación metodológica que permitió explorar las potencialidades de esta fuente de información relativamente nueva en el campo de la Geografía Urbana. Con la aplicación del Software QGIS se procesaron imágenes nocturnas de la Tierra provenientes del satélite Suomi NPP-VIIRS. Se identificó un crecimiento de la superficie de luminosidad urbana de un 13,87\% en un intervalo de 7 años. Por otro parte, se realizó una aproximación al conocimiento de la intensidad de los usos urbanos y cómo evolucionó durante el periodo estudiado, reflejando un incremento en la intensidad lumínica media de $17,77 \%$ para todo el conjunto de unidades político-administrativas del GBA. De igual manera, se evaluó la densidad de las luces nocturnas en las 25 unidades de análisis y se constató la hipótesis de que a mayor densidad de iluminación, mayor densidad poblacional con un coeficiente de correlación $r=0,87$. Esta primera aproximación metodológica permitió constatar a través del caso del GBA la utilidad y potencialidad de las imágenes nocturnas de la Tierra provenientes del satélite Suomi NPP-VIIRS, demostrando una gran cantidad de posibles aplicaciones en el campo de la Geografía científica.

\section{A B S T R A C T}

This paperwork aimed to analyze the urban expansion and density of Greater Buenos Aires (GBA) during the period 2012 - 2019, based on Earth's nocturnal satellite images. It was made a first methodological approach that allowed exploring the potential of this relatively new source of information in the urban geography field. There were processed night images of the Earth from the Suomi NPP-VIIRS satellite within the application of the QGIS Software. It was identified a growth of the surface of urban luminosity of $13.87 \%$ in a period of 7 years. On the other hand, it was made an approach to the knowledge of the intensity of urban uses and how it evolved during the studied period, reflecting an increase in the average light intensity of $17.77 \%$ for the whole set of politicaladministrative units of the GBA. Likewise, it was evaluated the density of the night lights in the 25 units of analysis and it was confirmed the hypothesis that higher density of illumination, greater the population density with a correlation coefficient $r=0.87$. This first methodological approach made it possible to verify, through the GBA case, the usefulness and potential of the night images of the Earth from the Suomi NPP-VIIRS satellite, demonstrating the large of possible applications in the field of scientific Geography. 


\section{Introducción}

En el marco de la Geografía como disciplina científica y en este caso, más específicamente de la Geografía Urbana, es posible observar cómo los métodos y técnicas que se aplican al estudio del espacio urbanizado han evolucionado a la par de los avances geotecnológicos. La Geografía, no es ajena a la actual revolución tecnológica e informacional del presente, ya que forma parte activa de la misma, especialmente en lo referente a la generación, acceso y manejo de Información Geográfica digital (Montes Galbán, 2017).

El uso creciente de las Tecnologías de la Información Geográfica (TIG) es cada vez mayor en la Geografía científica y profesional. Los Sistemas de Información Geográfica (SIG), la Cartografía Digital (CD), los Sistemas Globales de Navegación por Satélite (GNSS) y el Procesamiento Digital de Imágenes de satélite (PDI) son herramientas que tienen un gran impacto en los estudios geográficos actuales, permitiendo realizar investigaciones con mayor cobertura territorial, mayor precisión y mejora en los tiempos de captura y procesamiento de datos, llegando en algunos casos a la generación de información en tiempo real y cuasi-real (Montes Galbán, 2017). La aplicación de los mencionados avances al campo de la Geografía urbana ha sido totalmente posible puesto que esta, tiene como hilo conductor el enfoque espacial, haciendo especial referencia a la forma urbana (morfología), a su funcionalidad y las transformaciones históricas (Aguilera Arilla, Borderías Uribeondo, González Yanci, \& Santos Preciado, 1991).

El objetivo del presente trabajo consistió en analizar la expansión y densificación urbana del Gran Buenos Aires (GBA) para el periodo comprendido entre 2012 - 2019, a partir de las imágenes satelitales nocturnas de la Tierra. Se realizó una primera aproximación metodológica que permitió explorar las potencialidades de esta fuente de información relativamente nueva en tres líneas de análisis. Primero, se buscó determinar la extensión y morfología urbana del GBA; segundo, identificar la intensidad lumínica urbana en términos de su distribución espacial y temporal; por último, se estableció la relación entre la densidad de la luminosidad urbana y la densidad poblacional.

\section{Revisión metodológica}

Cuando se efectúa una primera revisión documental del uso de imágenes satelitales nocturnas en Geografía, es posible toparse en primera instancia con algunas publicaciones de divulgación científica general, que desde hace más de una década y media muestran su aplicación en proyectos de investigación a nivel planetario, este es el caso del mapa publicado por la National Geographic (2004) bajo el título "la Tierra de noche". Este mosaico del planeta se construyó en base a datos satelitales de luces nocturnas reunidos a lo largo de un año, en el cual se pueden identificar las regiones del planeta que poseen áreas urbanas con un brillo de luz blanca intensa, por otro lado, también destacan los grandes incendios, las locaciones con combustiones de gas y la pesca nocturna.

De igual manera, está el "Proyecto Huella Humana" desarrollado por la Wildife Conservation Society (WCS) y el Center for International Earth Science Information Network (CIESIN), su resultado permitió representar en un mapa global los niveles de influencia del ser humano en el planeta (especialmente sobre la vida salvaje y tierras vírgenes), uno de los factores considerados para la construcción de este mapa, fueron las redes de energía, que se midieron a través de las imágenes satelitales que perciben luces por la noche, puesto que es un indicador adecuado para la identificación de la infraestructura energética (National Geographic, 2005).

Las investigaciones que utilizan imágenes satelitales nocturnas ${ }^{1}$ y que buscan dar cuenta de las actividades humanas son cada vez más abundantes y diversas. Los trabajos comprenden temas como: estimación de los niveles de desarrollo y del producto interno bruto a escala subnacional (Elvidge C. D., Baugh, Anderson, Sutton, \& Ghosh, 2012²; Chen \& Nordhaus, 2010; Sutton, Elvidge, \& Ghosh, 2007), contaminación lumínica y sus efectos sobre la vida (Klinkenboborg, 2008) ${ }^{3}$, niveles de coincidencia territorial entre intensidades de la luz nocturna y la tasa de incidencia de enfermedades (Kloog, Haim,

1 Desde hace más de cuatro décadas se vienen recopilando imágenes globales nocturnas tomadas por los satélites DMSP (Elvidge et al. 2012) del Programa de Satélites Meteorológicos del Ministerio de Defensa de los EE.UU. (Defense Meteorological Satellite Program) a través de los sensores denominados OLS (Operational Linescan System - Sistema Operacional en Línea) cuyo propósito original era observar las nubes utilizando como medio la luz de la Luna y que, derivó posteriormente su aplicación en la determinación de las luces permanentes sobre la superficie de la Tierra.

2 La investigación generó como resultado un índice que permite estimar los niveles de desarrollo económico de los países a partir de la distribución espacial de la luz nocturna y la densidad de población.

3 En estos trabajos se analiza la eficiencia de la iluminación artificial y, se mide como las luces nocturnas se extienden sobre la naturaleza generando efectos directos sobre la vida (migración, reproducción y alimentación). 
Stevens, Barchana, \& Portnov, 2008) ${ }^{4}$, análisis de aspectos vinculados con el proceso de electrificación de los territorios (Sánchez de Miguel, Zamorano, Gómez Castaño, \& Pascual, 2014; Min, Gaba, Sarr, \& Agalassou, 2013; Townsend \& Bruce, 2010), estimar la distribución de la población a partir de datos de las luces nocturnas (Matayeva, 2014), análisis espacial sobre los conflictos armados (Li, Zhang, Huang, \& Li, 2015; Li \& Li, 2014).

Entre los trabajos que utilizan las imágenes satelitales nocturnas con la finalidad de estudiar los cambios espacio-temporales de las ciudades y lo relativo a los usos ubanos del territorio, estan los aportes de Henderson et al., (2003) en el cual ya se mencionaban las difultades metodológicas que se presentan al tratar de establecer un umbral de intensidad lumínica que permita definir las áreas urbanas. Para superar estas limitaciones se desarrollaron investigaciones que buscan la validación de los resultados obtenidos de las imágenes satelitales nocturnas comparandolo con otras fuentes satelitales (Small, Pozzi, \& Elvidge, 2005; Potere, Schneider, Angel, \& Civco, 2009). A pesar de las complejidades y limitaciones metodológicas se continua avanzando en el tema para la identificación, delimitación y monitoreo del crecimiento de las áreas urbanas a partir de las imágenes nocturnas, incrementandose los aportes científicos provenientes de diferentes continentes, América (Mitchell, 2001; Amaral, Monteiro, Camara, \& Quintanilha, 2006; Pérez Machado, Vizeu Barrozo, Luchiari, \& Small, 2014; Martin Oriol, 2016), Asia (Roy Chowdhury, Maithani, \& Dadhwal, 2012; Liu \& Leung, 2015; Xin, et al., 2017; Kamarajugedda, Mandapaka, \& Lo, 2017), Europa (Nel·lo, López Redondo, Martín Oriol, \& Checa Rius, 2016; Nel·lo, López, Martín, \& Checa, 2017; Checa, Gomá, López, Martín, \& Nel·lo, 2017; Checa \& Nel·lo, 2018) y estudios comparativos entre regiones del planeta (Zhou et al., 2015).

Los antecedentes de la referida temática en Argentina y en el área que atañe a la presente investigación no son abundantes. Se puede mencionar una propuesta metodológica a escala nacional que tuvo como objetivo identificar las áreas intermedias entre los usos urbano y rurales, con este fin se consideraron entre los materiales las imágenes satelitales nocturnas (Quaíni, 2014); un antecedente más reciente es el trabajo desarrollado por Martin Oriol (2016), quien realizó un estudio comparativo entre las urbes de Buenos Aires y Lima, analizando los cambios en la forma urbana a través de imágenes nocturnas de satélite; por último, desarrollamos una primera aproximación utilizando imágenes del satélite Suomi NPP (compuesto anual 2012) ofrecidas por la National Oceanic and Atmospheric Administration (NOAA, 2019) para estimar el porcentaje de superficie iluminada en los partidos de la cuenca del río Luján (Montes Galbán, 2019).

\section{Materiales y métodos}

\subsection{Delimitación espacial y temporal}

El área de estudio corresponde al conjunto urbano que el Instituto Nacional de Estadística y Censos denomina Gran Buenos Aires (GBA de aquí en adelante) ${ }^{5}$, el cual se conforma por la Ciudad de Buenos Aires y 24 partidos completos (Figura 1). Las 25 unidades político-administrativas suman para el año 2010 una población total de 18.745.423 habitantes (INDEC, 2019). Como el objetivo general de la presente investigación se centra en identificar los usos urbanos del territorio, se decidió excluir del análisis el territorio de islas correspondiente al partido San Fernando, por ser una zona con poca población y sin áreas urbanizadas (Figura 1). En cuanto al espacio físico, quedó definida una superficie total de 2.997,82 km².

\footnotetext{
$4 \quad$ Partiendo de los datos de luminosidad nocturna y a través de una regresión múltiple fue posible constatar que hay $73 \%$ más de incidencia de cáncer de mama en aquellas comunidades con mayor exposición a la luz nocturna en Israel.

5 "El INDEC denominará así al área comprendida por la Ciudad de Buenos Aires más los Partidos del Gran Buenos Aires (en el sentido administrativo, es decir, 24 partidos completos). Esta es la denominación que vienen utilizando la mayoría de los programas de trabajo del INDEC, tanto en el área económica como social...” (INDEC, 2003, p. 4).
} 
Figura 1. Área de estudio y unidades político-administrativas del Gran Buenos Aires.

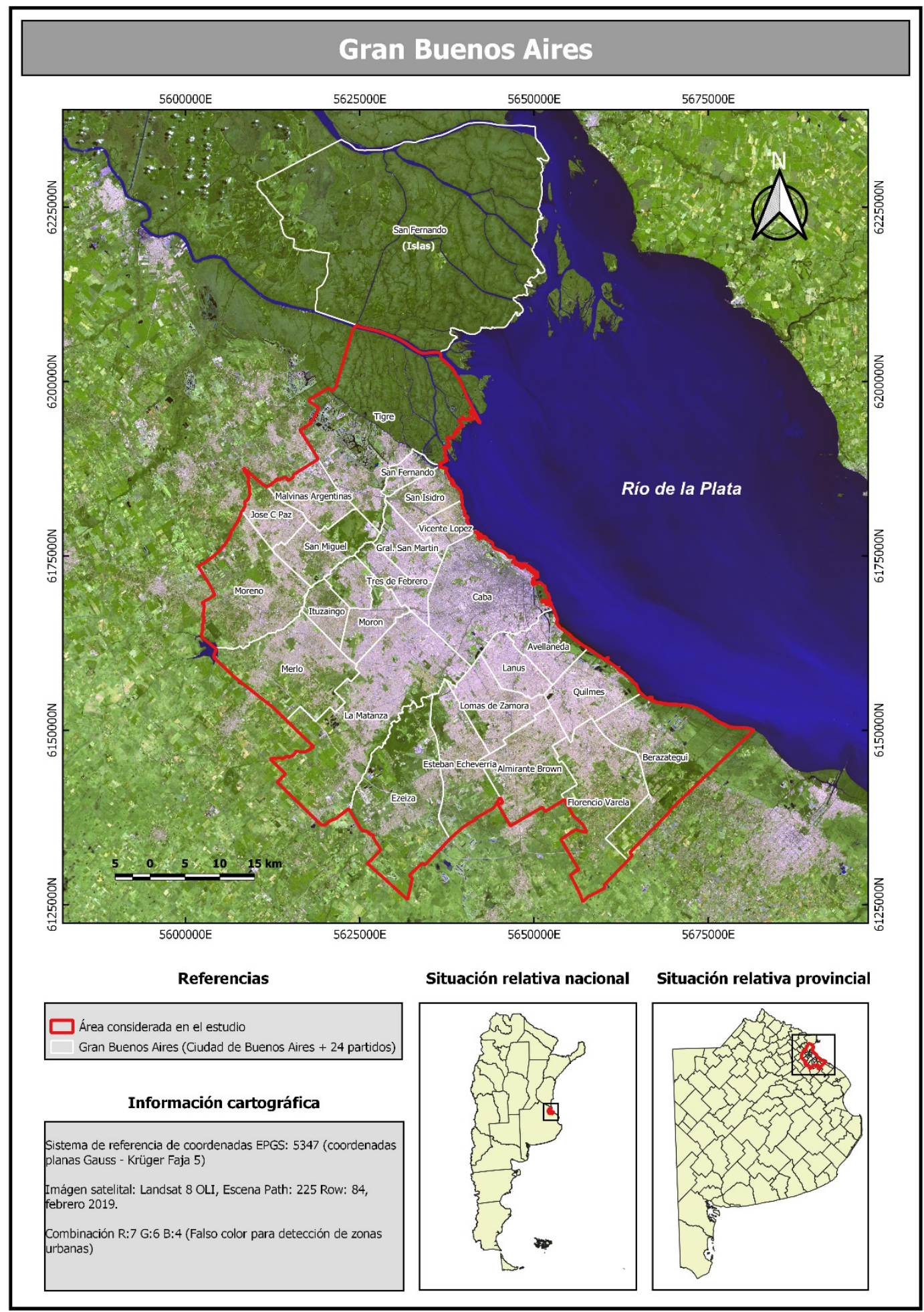

Fuente: elaboración propia en base a datos del Instituto Geográfico Nacional (2019), United States Geological Survey (2019) y el Instituto Nacional de Estadísticas y Censos (2003).

Desde un punto de vista socio-espacial, el GBA se caracteriza por tener la complejidad de las grandes ciudades, al respecto Buzai y Marcos afirman que "las condiciones socioeconómicas favorables decaen claramente desde el centro hacia la periferia en configuraciones de anillos y sectores según sean las características de fricción espacial y, en amplias zonas periféricas, aparecen abruptamente elementos puntuales de alto nivel propios de la ciudad de islas..." (2012, p. 74).

La investigación tuvo un alcance temporal con un intervalo de siete años, definido para el periodo 2012 - 2019, específicamente los meses de abril de los mencionados años, este periodo de tiempo seleccionado obedece principalmente a la disponibilidad de datos al momento de realizar el estudio. 


\subsection{Información básica}

\subsubsection{Fuente de los datos satelitales}

Los datos primarios utilizados en la investigación están conformados por las imágenes de la Tierra por la noche producidas por el Grupo de Observaciones de la Tierra (Earth Observation Group - EOG por sus siglas en inglés), que forma parte del Centro Nacional de Datos Geofísicos (NGDC por sus siglas en inglés) de la Administración Nacional Oceánica y Atmosférica de los Estados Unidos (NOAA por sus siglas en inglés). Dichas imágenes son una composición que es posible gracias a la banda día/noche (DNB) del instrumento VIIRS (Serie de radiómetros de imágenes infrarrojas visibles) a bordo del satélite Suomi National Polar Partnership (Suomi NPP) ${ }^{6}$. Parte de los mosaicos globales que se utilizaron en la investigación como datos primarios nocturnos corresponden al conjunto de imágenes compuestas de radiancia promedio captadas por el VIIRS?.

Los datos que presenta el EOG disponibles para su descarga en la versión $1^{8}$, tienen una cobertura planetaria que va desde los $75^{\circ}$ de latitud Norte hasta los $65^{\circ}$ de latitud Sur. Los productos se generan en cuadrículas geográficas de 15 segundos de arco (aproximadamente 380 x $380 \mathrm{~m}$. en la latitud correspondiente al área de estudio) y están disponibles en formato GeoTIFF. El planeta está dividido en 6 grandes mosaicos con una serie temporal (para el momento de la investigación) que va desde abril de 2012 hasta abril de 2019 (Tabla 1). Para los efectos de la presente aplicación se trabajó con el cuadrante $\mathrm{N}^{\circ}$ 5 dentro del cual está contenida el área de interés.

Tabla 1. Características del VIIRS-DNB y las imágenes utilizadas.

\begin{tabular}{|c|c|}
\hline Características & Descripción \\
\hline Cuantificación radiométrica & 14 bits / 16.384 DN \\
\hline Valores de luminosidad de los píxeles & $\begin{array}{c}\text { 0 a 300 nanoWatts/cm2.sr (Rango de valores } \\
\text { normales) }\end{array}$ \\
\hline Saturación & Sin saturación \\
\hline Tamaño de pixel & 15 segundos de arco \\
\hline Calibración radiométrica & Calibrador a bordo \\
\hline Bandas espectrales & 11 de noche/ 21 de día \\
\hline Sensibilidad a la radiación & Pancromática $500-900 \mathrm{~nm}$ \\
\hline Hora nocturna de paso & $01: 30 \mathrm{~h}$ UTC \\
\hline Cobertura de las imágenes descargadas & $00 \mathrm{~N} / 060 \mathrm{~W}$ \\
\hline Serie temporal (mes-año) & Abril 2012 y abril 2019 \\
\hline
\end{tabular}

Fuente: elaboración propia en base a Elvidge et al., (2013) y Checa y Nel·lo (2018).

El valor de los píxeles en este caso representa el nivel medio de la radiación emitida por la superficie, presentándose en unidades de nanoWatts $/ \mathrm{cm}^{2} . \mathrm{sr}$, con un rango de valores normal que se encuentra entre 0 y 300 nanoWatts/cm2.sr, indicando el 0 la mayor oscuridad y el 300 la intensidad de máxima luz 9 .

$6 \quad$ El Suomi NPP es el resultado de una misión conjunta entre la Administración Nacional de Aeronáutica y del Espacio (NASA, por sus siglas en inglés) con el apoyo operacional de la Administración Nacional Oceánica y Atmosférica (NOAA por sus siglas en inglés) y su sistema conjunto de satélites polares. La vista nocturna de la superficie terrestre es posible gracias a la banda de día y noche de la Suite de Radiómetro de Imágenes Infrarrojas Visibles (VIIRS), el sensor detecta la luz en un rango de longitudes de onda de verde a infrarrojo cercano y utiliza técnicas de filtrado para observar señales débiles como bengalas de gas, auroras, incendios forestales, luces de la ciudad y la luz de la luna reflejada.

7 La creciente demanda de los productos de iluminación nocturna VIIRS por una amplia variedad de usuarios (científicos de uso del suelo, geógrafos urbanos, ecologistas, modelistas de carbono, astrónomos, demógrafos, economistas y científicos sociales) ha propiciado el desarrollo de algoritmos para la generación de productos de luces nocturnas globales de alta calidad (Elvidge C. D., Baugh, Zhizhin, Chi Hsu, \& Ghosh, 2017).

$8 \quad$ Entre la variedad de productos que el Grupo de Observaciones de la Tierra pone a disposición para su descarga están los promedios temporales de forma mensual y anual (https://www.ngdc.noaa.gov/eog/viirs/download_dnb_composites.html). La versión 1 de la serie de compuestos mensuales no están filtradas, ya que buscan proteger las luces de auroras, incendios, barcos y otras luces temporales. Sin embargo, los compuestos anuales tienen capas con separación adicional, eliminando luces temporales y valores de fondo (no luminosos).

$9 \quad$ En este rango de valores es posible distinguir los asentamientos pequeños y también los valores de radiancia (variaciones intraurbanas) sin saturaciones en las áreas con más luz (Small, Elvidge, \& Baugh, 2013). 


\subsubsection{Datos en formato vectorial}

Para el logro de los objetivos planteados fue necesario descargar los siguientes datos en formato shapefile (.SHP) provenientes del banco de datos abiertos del Instituto Geográfico Nacional (IGN, 2019):

- Coberturas del suelo (2013)

- Departamentos/ Partidos

- Provincias

\subsection{Tratamiento de la información}

En esta etapa de la investigación fueron tenidos como referencia los trabajos desarrollados por el Grupo de investigación sobre Energía, Territorio y Sociedad de la Universitat Autónoma de Barcelona (Nel·lo et al., 2016; Checa et al., 2017; Checa \& Nel·lo, 2018).

Una vez descargados los datos, se procedió en el software QGIS 3.4.12 a configurar el proyecto bajo el EPSG: 5347, POSGAR 2007 / Argentina 5, esto permitió tener toda la base de datos espacial unificada bajo un mismo sistema de referencia de coordenadas planas y facilitó los posteriores cálculos de superficie y distancias.

El paso siguiente fue realizar el recorte de las imágenes a la correspondiente área de estudio (GBA) para lo cual fue necesario utilizar una capa vectorial como mascara.

Seguidamente, se sometieron las imágenes seleccionadas a una revisión y filtrado para poder compensar cualquier distorsión generada por "luces temporales". Con el objeto de contrarrestar las posibles anomalías generadas por incendios u otras fuentes de luces eventuales fue necesario corregir las imágenes calculando el valor promedio para los meses de estudio en años posteriores (en el caso del 2012) y anteriores (en el caso del 2019) y así asignar el dato a los píxeles afectados.

Como todos los objetivos de la investigación giran en torno al conocimiento de la extensión de la superficie urbanizada a partir de la superficie iluminada y, como no todos los píxeles con valores mayores que cero corresponden a las áreas de usos urbanos de la tierra, se tuvo que estimar un valor mínimo (intensidad lumínica) de referencia. Esto permitió definir el valor mínimo de luz en la que un área de superficie iluminada corresponde más estrechamente a la presencia de construcciones, infraestructuras y otros artefactos (Checa \& Nel·lo, 2018) ${ }^{10}$. Para concretar este paso, se superpuso la capa con la cobertura urbano-industrial 2013 del Instituto Geográfico Nacional sobre la imagen 2012, luego se realizó un proceso de extracción de los valores mínimos (Estadísticas de zona) y posteriormente se promediaron para llegar a establecer el umbral de luz urbana en 23,91 nanoWatts/cm2.sr.

A partir del valor de referencia se delimitó la superficie de luminosidad urbana en las imágenes 2012 y 2019, tomandose en consideración aquellos píxeles que tuvieran valores $\geq 23,91$ nanoWatts $/ \mathrm{cm} 2$. sr. (Figuras 2 y 3 ).

\footnotetext{
$10 \quad$ Esto no quiere decir, que los usos urbanos no se puedan extender en aquellas áreas con niveles de luz por debajo del valor de referencia establecido, como aclara Checa \& Nel-lo (2018) el concepto de luz urbana debe entenderse en terminos de intensidad más que de exclusión.
} 
Figura 2. Imágenes del satélite VIIRS / DNB y delimitación del área de estudio, abril 2012 - 2019.

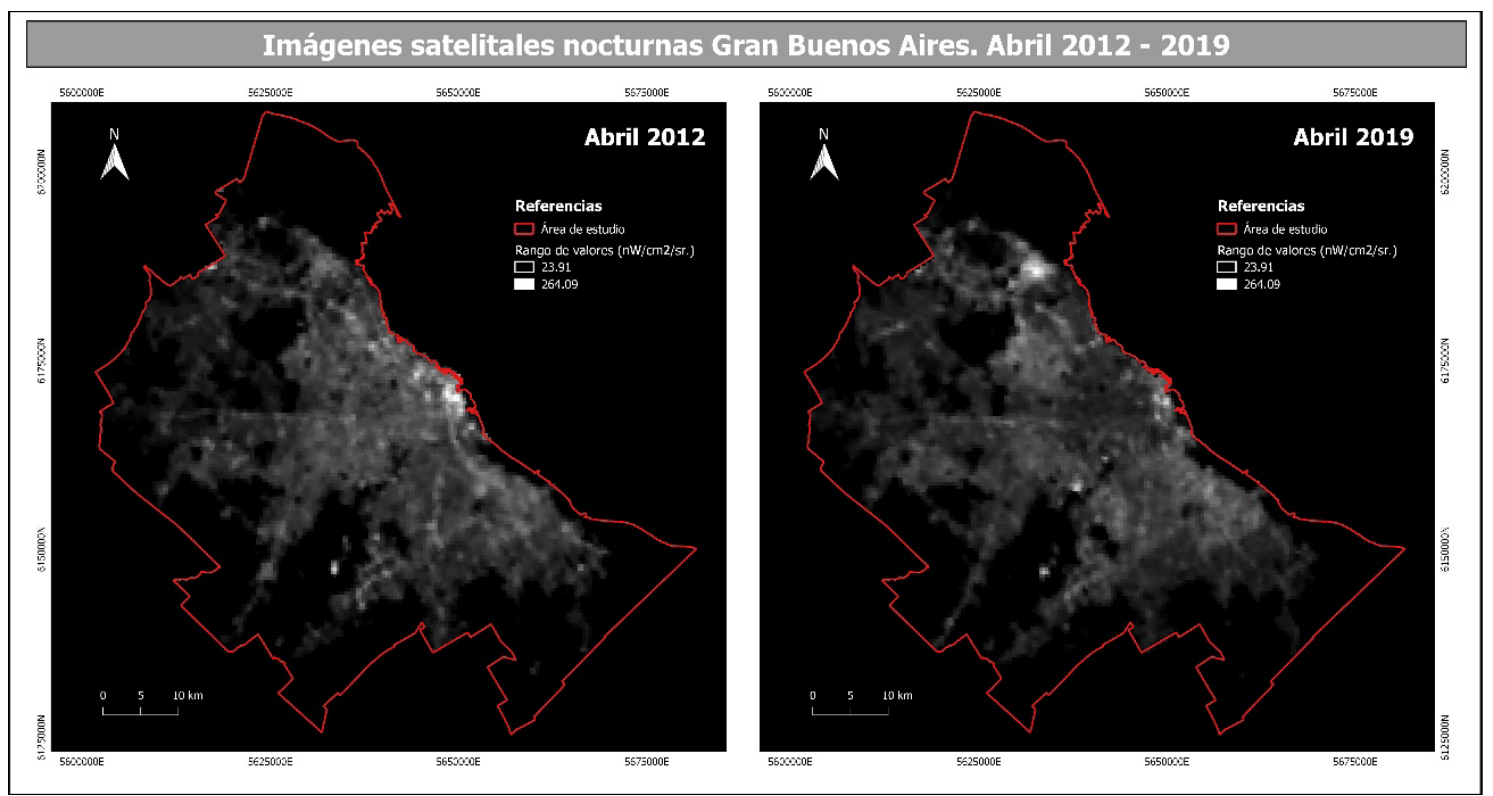

Fuente: elaboración propia en base a datos de la National Oceanic and Atmospheric Administration (2019) y el Instituto Geográfico Nacional (2019).

Figura 3. Imágenes del satélite VIIRS / DNB y unidades político-administrativas del GBA, abril 2012 - 2019.

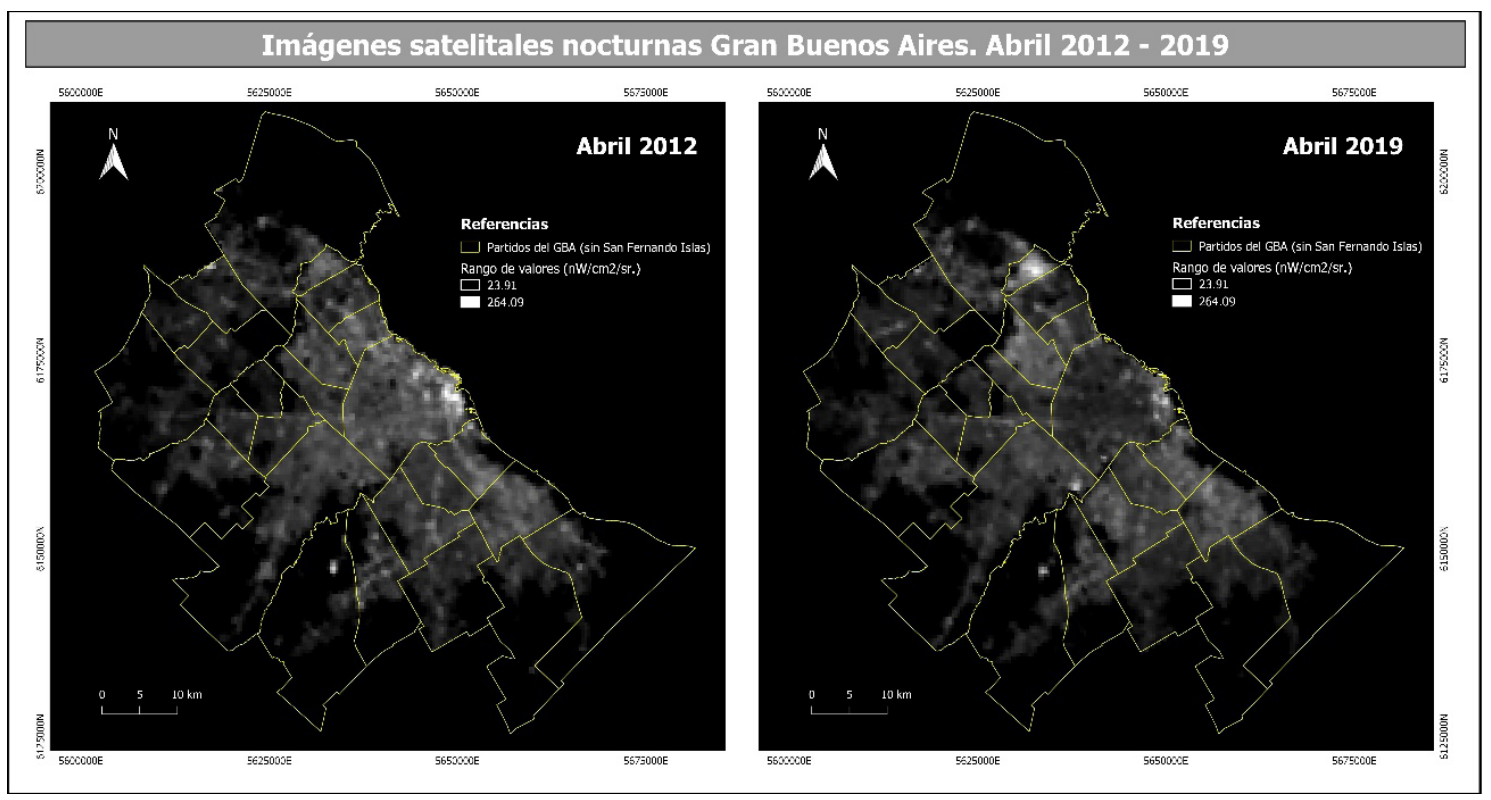

Fuente: elaboración propia en base a datos de la National Oceanic and Atmospheric Administration (2019) y el Instituto Geográfico Nacional (2019).

Las imágenes resultantes de los procesos anteriores permitieron derivar los principales resultados de la investigación. Primero, se vectorizaron ambas imágenes para luego calcular la superficie $\left(\mathrm{km}^{2}\right)$ de luminosidad urbana 2012-2019. Segundo, se extrajeron los valores promedio de luminosidad para cada una de las unidades político-administrativas del GBA 2012-2019, esto a su vez, permitió generar salidas en forma de mapas y tablas con las estadísticas descriptivas. Tercero y último, se aplicó la fórmula propuesta por Matayeva (2014, p.25) para definir la densidad de la luz nocturna:

$$
\lambda=\mathrm{L} / \mathrm{A}
$$

Fórmula 1. Densidad de luz nocturna.

Donde, - densidad de luz nocturna, nano Watts $/\left(\mathrm{cm}^{2} \cdot \mathrm{sr} \cdot \mathrm{km}^{2}\right)$, - intensidad de luz nocturna, nano Watts $/\left(\mathrm{cm}^{2} \cdot \mathrm{sr}\right)$, - área de una unidad administrativa, $\mathrm{km}^{2}$.

Este resultado a su vez posibilitó realizar los gráficos y mapas de correlación entre la densidad de 
las luces nocturnas vs densidad de población ${ }^{11}$. La secuencia de pasos metodológico-técnico se encuentran explicitados en el diagrama de flujo de la Figura 4.

Figura 4. Diagrama de flujo de procedimientos.

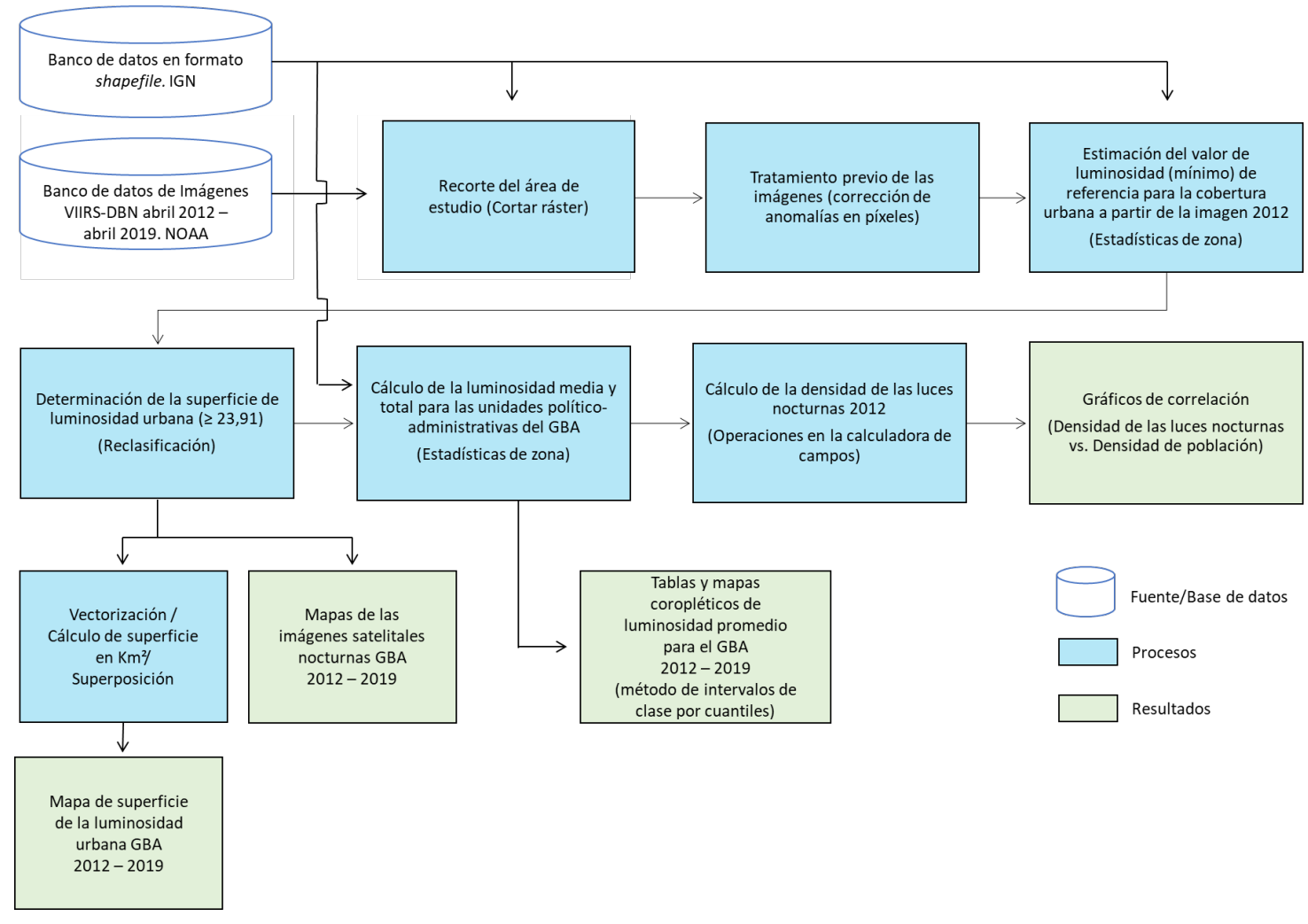

Fuente: elaboración propia.

\section{Resultados}

Los resultados del análisis de la expansión y densificación urbana del GBA se presentan en función de tres objetivos específicos. En primer lugar, se determinó la extensión y morfología urbana del GBA (2012-2019) a partir de las imágenes satelitales nocturnas. En segundo lugar, se identificó la intensidad lumínica urbana en términos de su distribución espacial y temporal (2012-2019). Por último, se estableció la relación entre la densidad de la luminosidad urbana y la densidad poblacional para el año 2012 por ser este el dato analizado más cercano a las estadísticas del último censo.

\subsection{Extensión y morfología de los usos urbanos a partir de las imágenes satelitales nocturnas}

En la figura 5, se puede observar la superficie que presentó niveles de luz $\geq 23,91$ y que, hasta el año 2012 era de 1.695,5 $\mathrm{Km}^{2}$ mientras que, en el 2019 fue de 1.930,82 $\mathrm{Km}^{2}$, presentando un incremento de un 13,87\% en el intervalo de 7 años.

Además del calculo de la superficie, el mapa de la Figura 5 permite observar y analizar las formas adoptadas por las áreas urbanas en su proceso de expansión, en este caso, si se compara con los resultados del mapa social elaborado por Buzai y Marcos (2012) es posible verificar que en la actualidad se mantienen algunos patrones de crecimiento para el GBA, como las configuraciones de anillos y sectores, así como el surgimiento de pequeñas áreas en forma de "archipiélagos" urbanos. 
Figura 5. Superficie de luminosidad urbana ( $\geq 23,91)$, abril (2012-2019).

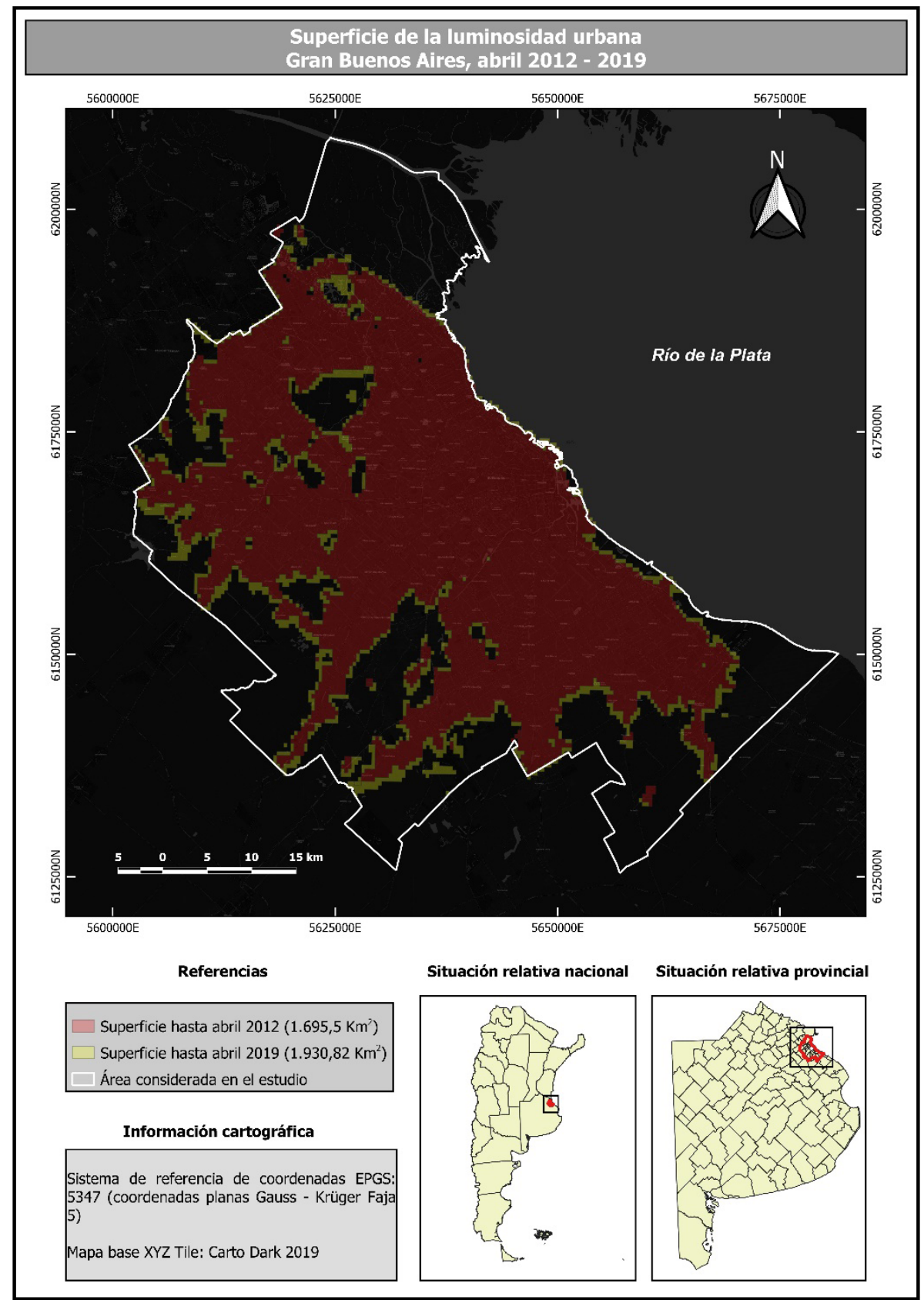

Fuente: Elaboración en base a cálculos propios.

\subsection{Distribución espacio-temporal de la intensidad lumínica urbana}

El cálculo de intensidad lumínica media por cada unidad político-administrativa (25 en total) permite ampliar el análisis realizado hasta ahora, porque genera una noción sobre la intensidad de los usos urbanos del territorio, permitiendo conocer aquellas áreas que mantienen, disminuyen o aumentan las intensidades. A partir de la Figura 6 y Tabla 2, se puede observar varios cambios para el intervalo de 7 años, el $88 \%$ del territorio estudiado tuvo un incremento de la luminosidad media, solo tres de las unidades político-administrativas presentaron una disminución de la intensidad lumínica (valores negativos en la Tabla 2). 
Figura 6. Luminosidad media por unidad administrativa del GBA, meses de abril (2012-2019).

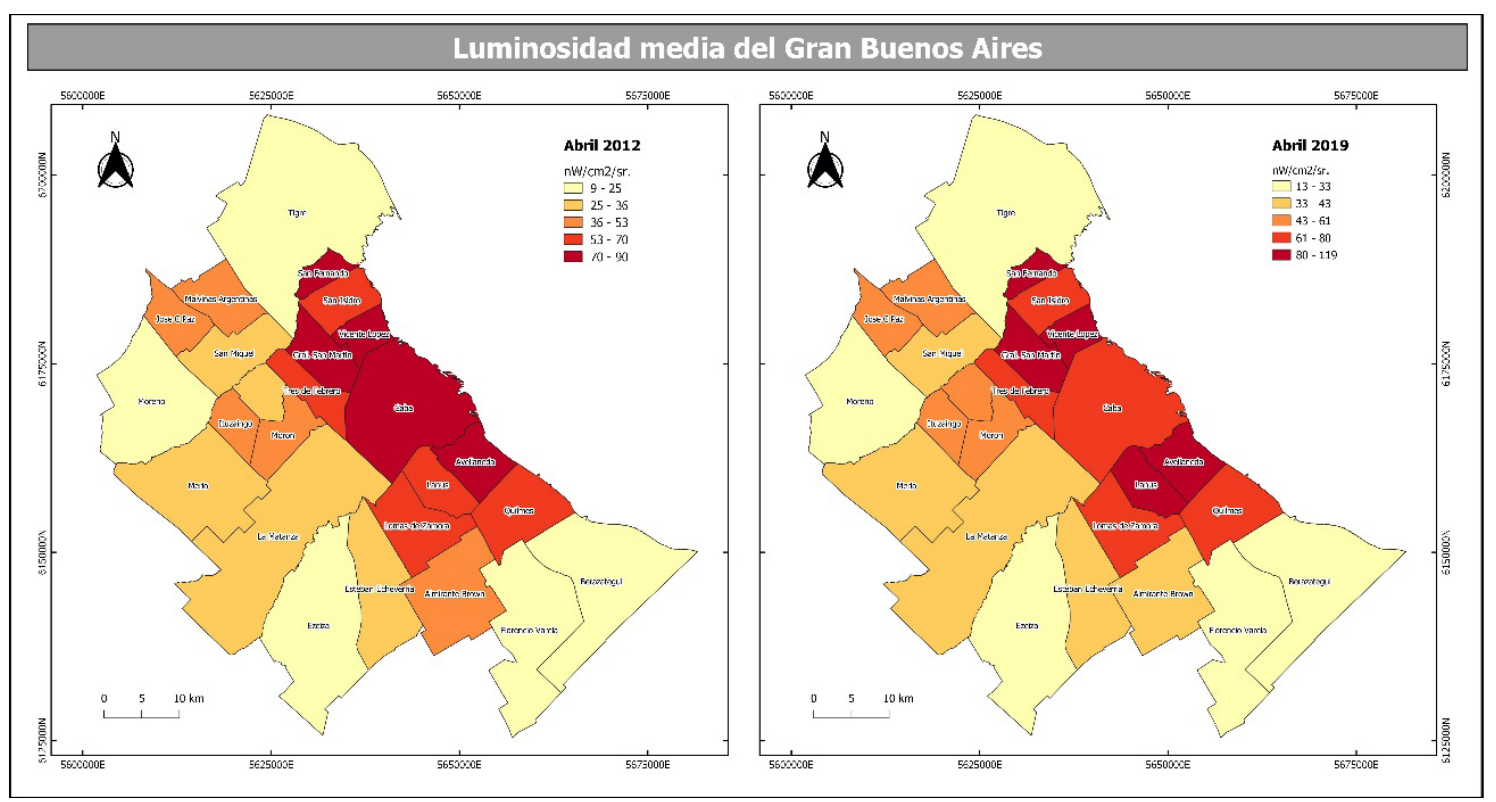

Fuente: elaboración en base a cálculos propios.

Al evaluar las estadísticas descriptivas generales, se puede evidenciar que tanto los valores mínimos como máximos tuvieron un incremento importante, un 39,08\% y 33,13\% respectivamente. La media de todos los datos aumentó un 17,77\% y, la desviación estándar un 13,03\%, este último valor nos permite concluir que las diferencias territoriales incrementaron.

Tabla 2. Valores promedio de luminosidad y porcentaje de cambio por unidad político-administrativa del GBA, meses de abril (2012-2019).

\begin{tabular}{|c|c|c|c|}
\hline \multirow{2}{*}{$\begin{array}{l}\text { Unidades político- } \\
\text { administrativas }\end{array}$} & \multicolumn{2}{|c|}{$\begin{array}{c}\text { Media } \\
\text { (nanoWatts/cm2.sr) }\end{array}$} & \multirow{2}{*}{ Cambio relativo (\%) } \\
\hline & 2012 & 2019 & \\
\hline San Fernando & 76.88 & 119.46 & 55.39 \\
\hline Gral. San Martin & 70.73 & 96.35 & 36.23 \\
\hline Vicente López & 89.34 & 83.93 & -6.05 \\
\hline Avellaneda & 72.44 & 82.95 & 14.50 \\
\hline Lanús & 67.61 & 80.43 & 18.95 \\
\hline Quilmes & 70.18 & 79.93 & 13.89 \\
\hline Lomas de Zamora & 63.92 & 79.70 & 24.69 \\
\hline CABA & 89.74 & 70.74 & -21.17 \\
\hline San Isidro & 62.92 & 67.98 & 8.03 \\
\hline Tres de Febrero & 57.22 & 65.88 & 15.13 \\
\hline Morón & 49.83 & 58.07 & 16.53 \\
\hline Ituzaingó & 48.80 & 56.69 & 16.17 \\
\hline Malvinas Argentinas & 48.19 & 51.97 & 7.84 \\
\hline José C. Paz & 36.16 & 50.61 & 39.93 \\
\hline Hurlingham & 31.03 & 44.86 & 44.57 \\
\hline La Matanza & 35.99 & 41.22 & 14.53 \\
\hline Almirante Brown & 36.82 & 40.38 & 9.66 \\
\hline Esteban Echeverria & 31.18 & 37.61 & 20.62 \\
\hline Merlo & 25.44 & 36.45 & 43.31 \\
\hline San Miguel & 25.68 & 33.49 & 30.40 \\
\hline Moreno & 20.92 & 29.35 & 40.28 \\
\hline Tigre & 14.96 & 18.99 & 26.98 \\
\hline Florencio Varela & 15.15 & 18.67 & 23.21 \\
\hline Berazategui & 18.36 & 17.96 & -2.19 \\
\hline
\end{tabular}




\begin{tabular}{|c|c|c|c|}
\hline Ezeiza & 9.01 & 12.53 & 39.08 \\
\hline Mínimo & 9.01 & 12.53 & 39.08 \\
\hline Máximo & 89.74 & 119.46 & 33.13 \\
\hline Media & 46.74 & 55.05 & 17.77 \\
\hline Desviación estándar & 24.23 & 27.39 & 13.03 \\
\hline
\end{tabular}

Fuente: Elaboración en base a cálculos propios.

\subsection{Densidad de la luminosidad urbana y densidad de población}

En la Figura 7, se observa un gráfico de dispersión con la relación entre la densidad de la luminosidad urbana y la densidad de población, se corrobora que existe una alta asociación positiva con un coeficiente de correlación $r=0,87$ entre ambas distribuciones espaciales, lo cual indica que de acuerdo con el coeficiente $\mathrm{r}^{2}$ una variable explica el $76 \%$ del comportamiento de la otra, es decir que para este caso, sería posible estimar la distribución de la densidad de la población a partir de la luminosidad nocturna.

Figura 7. Correlación entre la densidad de las luces nocturnas vs. densidad de población.

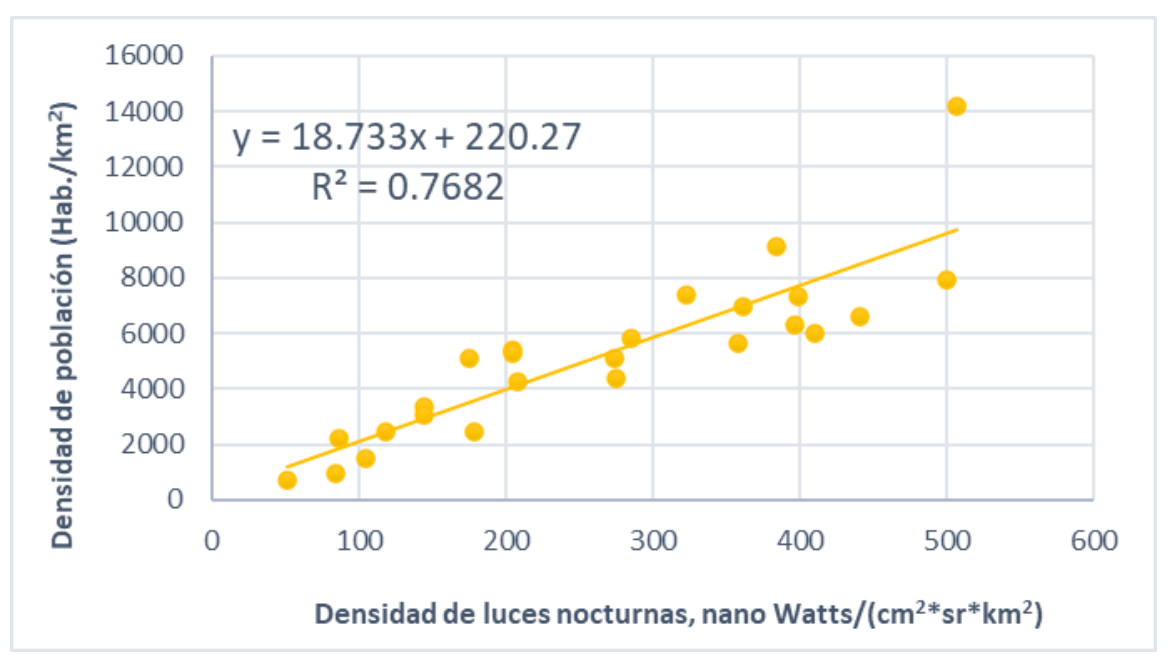

Fuente: elaboración en base a cálculos propios.

El resultado que se presenta en la Figura 8, permite profundizar el análisis del comportamiento espacial de la relación antes establecida entre densidad de las luces nocturnas y densidad poblacional, se constata como aquellas unidades espaciales centrales con menor superficie y altos niveles de población ubicadas dentro de lo que sería el primer y segundo anillo de crecimiento del GBA, se encuentran en el nivel de correlación del cuadrante superior-derecho, por otro lado, aquellas unidades espaciales con baja densidad lumínica y poblacional ubicadas dentro del tercer anillo de crecimiento del GBA, están en el nivel de relación del cuadrante inferior-izquierdo. 
Figura 8. Relación bivariada entre la densidad de las luces nocturnas y la densidad de población, la Figura 8A muestra las unidades espaciales con valores altos de densidad (cuadrante superior-derecho); la Figura 8B muestra las unidades espaciales con valores bajos de densidad (cuadrante inferior-izquierdo).
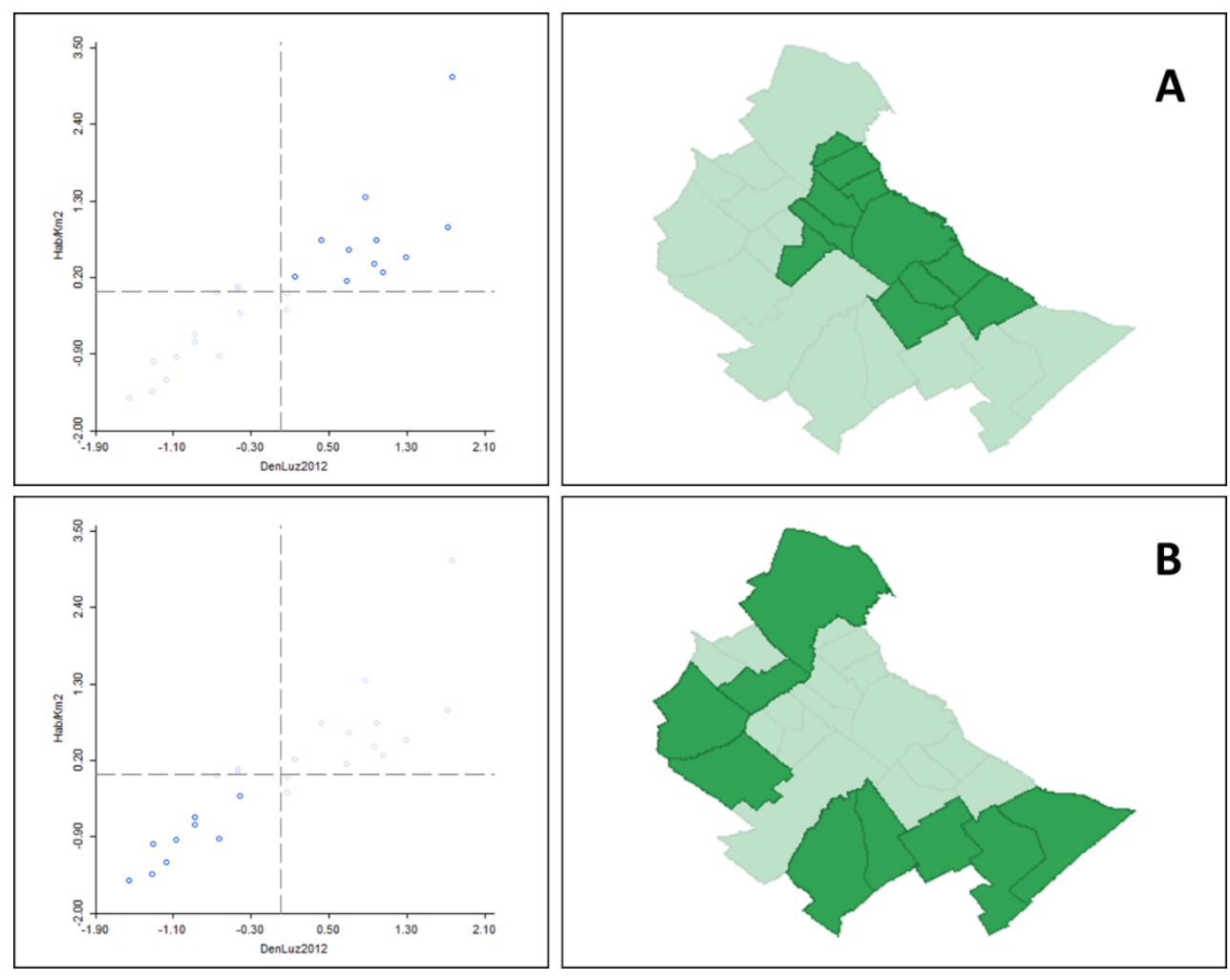

Elaboración en base a cálculos propios.

\section{Conclusiones}

Esta primera aproximación metodológica permitió constatar a través del caso del GBA la utilidad y potencialidad de las imágenes nocturnas de la Tierra provenientes del satélite Suomi NPP-VIIRS. Se pudo comprobar que a través de esta fuente de información relativamente nueva es posible analizar la extensión, morfología, intensidad y evolución de los usos urbanos del territorio, asimismo, evaluar la relación existente entre la iluminación y la distribución espacial de otras variables sociodemográficas.

El abordaje del área estudiada (GBA) a través de la superficie de luminosidad urbana en el periodo 2012-2019 permitió identificar un crecimiento de un 13,87\% en un intervalo de 7 años. Asimismo, fue posible verificar que en la actualidad se mantienen algunos patrones de crecimiento (anillos, sectores y pequeñas áreas en forma de "archipiélagos" urbanos).

Por otro parte, se realizó una aproximación al conocimiento de la intensidad de los usos urbanos y cómo evolucionó durante el periodo estudiado en el GBA, reflejando un incremento en la intensidad lumínica media de $17,77 \%$ para todo el conjunto de unidades político-administrativas del GBA. De igual manera, se evaluó la densidad de las luces nocturnas en las 25 unidades de análisis y se constató la hipótesis de que, a mayor densidad de iluminación, mayor densidad poblacional con un coeficiente de correlación $r=0,87$.

Las imágenes VIIRS-DNB presentan notables mejoras técnicas con respecto a sensores anteriores, permitiendo generar compuestos anuales y mensuales de luz nocturna con mejor precisión, resolución y calibración, abriendo un abanico de posibles aplicaciones dentro del campo de la Geografía científica. Los productos generados a partir de este tipo de imágenes (tomando en cuenta su resolución espacial) admite el desarrollo de cartografía a pequeña escala $(\leq 1: 700.000)$ que a su vez posibilita el análisis geográfico del fenómeno urbano con un alcance a escala regional.

En el caso particular de la Geografía Urbana este nuevo instrumento permite realizar mediciones del objeto de estudio que posteriormente facilitan la aproximación a definiciones espaciales actualizadas, analizando aspectos del crecimiento urbano tradicional como la ciudad compacta y la ciudad difusa. Todo lo mencionado anteriormente, convierte a las imágenes nocturnas de la Tierra en otra herramienta disponible para su uso en la planificación urbana y regional, así como un apoyo para la gestión de las metrópolis transjurisdiccionales. 


\section{Agradecimientos} trabajo.

El autor agradece al Dr. Gustavo Buzai por sus invaluables sugerencias en el desarrollo del presente

\section{Referencias bibliográficas}

Aguilera Arilla, J., Borderías Uribeondo, P., González Yanci, P., \& Santos Preciado, J. M. (1991). Geografía general II (Geografía humana). Madrid: Universidad Nacional de Educación a Distancia.

Amaral, S., Monteiro, A. M., Camara, G., \& Quintanilha, J. A. (2006). DMSP/OLS night time light imagery for urban population estimates in the Brazilian Amazon. International Journal of Remote Sensing, 27(5), 855-870. doi:10.1080/01431160500181861

Buzai, G. D., \& Marcos, M. (2012). The social map of greater Buenos Aires as empirical evidence of urban models. Journal of Latin American Geography, 11(1), 67-78.

Center for Spatial Data Science. The University of Chicago. (15 de Noviembre de 2019). Software of the Center for Spatial Data Science. Recuperado de https://spatial.uchicago.edu/software

Checa, J., \& Nel.lo, O. (2018). Urban Intensities. The Urbanization of the Iberian Mediterranean Coast in the Light of Nighttime Satellite Images of the Earth. Urban Science, 2(4), 1-17. doi:10.3390/ urbansci2040115

Checa, J., Gomà, A., López, J., Martín, J., \& Nel·lo, O. (2017). Energía y proceso de urbanización en España. Nuevas perspectivas de investigación a partir de la imagen satelital nocturna de la tierra. En M. Zaar, M. Vasconselos, \& H. Capel (Eds.), La electrificación y el territorio. Historia y futuro (pp. 1-20). Barcelona: Universitat de Barcelona. Recuperado el 1 de Julio de 2019, de http://www.ub.edu/geocrit/ Electr-y-territorio/ChecaGoma.pdf

Chen, X., \& Nordhaus, W. D. (2010). The value of luminosity data as a proxy for economic statistics. Nber working paper series, 1-43.

Elvidge, C. D., Baugh, K. E., Anderson, S. J., Sutton, P. C., \& Ghosh, T. (2012). The Night Light Development Index (NLDI): a spatially explicit measure of human development from satellite data. Social Geography, 7, 23-35. doi:10.5194/sg-7-23-2012

Elvidge, C. D., Baugh, K., Zhizhin, M., \& Chi Hsu, F. (2013). Why VIIRS data are superior to DMSP for mapping nighttime lights. Proceedings of the Asia-Pacific Advanced Network, 35, 62-69. doi:10.7125/ APAN.35.7

Elvidge, C. D., Baugh, K., Zhizhin, M., Chi Hsu, F., \& Ghosh, T. (2017). VIIRS night-time lights. International Journal of Remote Sensing, 38(21), 5860-5879. doi:10.1080/01431161.2017.1342050

Henderson, M., Yeh, E. T., Gong, P., Elvidge, C., \& Baugh, K. (2003). Validation of urban boundaries derived from global night-time satellite imagery. International Journal of Remote Sensing, 24(3), 595-609. doi:10.1080/01431160304982

Instituto Geográfico Nacional. (2019). Datos abiertos. Capas SIG. Recuperado de https://www.ign. gob.ar/NuestrasActividades/InformacionGeoespacial/CapasSIG

Instituto Nacional de Estadística y Censos. (1 de Noviembre de 2019). Estadísticas de población. Recuperado de https://www.indec.gob.ar/

Instituto Nacional de Estadística y Censos. (2003). ¿Qué es el Gran Buenos Aires? Buenos Aires: Publicaciones delINDEC.Recuperadoel15 deJuniode2019, dehttps://web.archive.org/web/20101113161116/ http://www.indec.gov.ar/nuevaweb/cuadros/4/folleto\%20GBA.pdf

Kamarajugedda, S. A., Mandapaka, P. V., \& Lo, E. Y. (2017). Assessing urban growth dynamics of major Southeast Asian cities using night-time light data. International Journal of Remote Sensing, 38(21), 6073-6093. doi: $10.1080 / 01431161.2017 .1346846$

Klinkenboborg, V. (Noviembre de 2008). Our vanishing night. National geographic, 214(5), 102-123.

Kloog, I., Haim, A., Stevens, R. G., Barchana, M., \& Portnov, B. A. (2008). Light at night co-distributes with incident breast but not lung cancer in the female population of Israel. Chronobiology International, 25(1), 65-81. doi:10.1080/07420520801921572

Li, X., \& Li, D. (2014). Can night-time light images play a role in evaluating the Syrian Crisis? 
International Journal of Remote Sensing, 35(18), 6648-6661. doi:10.1080/01431161.2014.971469

Li, X., Zhang, R., Huang, C., \& Li, D. (2015). Detecting 2014 Northern Iraq Insurgency using nighttime light imagery. International Journal of Remote Sensing, 36. doi:10.1080/01431161.2015.1059968

Liu, L., \& Leung, Y. (2015). A study of urban expansion of prefectural-level cities in South China using night-time light images. International Journal of Remote Sensing, 36, 5557-5575. doi:10.1080/014311 $\underline{61.2015 .1101650}$

Matayeva, A. (2014). Deriving the distribution of population from nighttime lights data (tesis de maestría). Technische Universitat Munchen, Munich.

Martin Oriol, J. (2016). Observing changes in urban form through night satellite images in Buenos Aires and Lima. En O. Nel·lo, \& R. Mele (Eds.), Cities in the 21st Century (págs. 93-95). London and New York: Routledge.

Min, B., Gaba, K. M., Sarr, O. F., \& Agalassou, A. (2013). Detection of rural electrification in Africa using DMSP-OLS night lights imagery. International Journal of Remote Sensing, 34(22), 8118-8141. doi:10.10 $\underline{\text { 80/01431161.2013.833358 }}$

Mitchell, J. G. (Julio de 2001). La expansión urbana. National geographic en español, 9(1), 82-107.

Montes Galbán, E. (2017). Ideas sobre tecnociencia y experimentación digital en geografía. Vientos del Norte, 2, 7-17. Recuperado el 1 de Enero de 2017, de http://editorial.unca.edu.ar/Publicacione\%20on\%20 line/CD\%20VIENTOS\%202017/PDF/N5\%20Vol\%20II/1-Ideas\%20sobre\%20tecnociencia.pdf

Montes Galbán, E. (2019). Cuenca. Luminosidad nocturna. En G. D. Buzai, \& S. L. Lanzelotti (Dir.), Atlas de Geografía Humana de la cuenca del río Luján (pp. 168-169). Ciudad Autónoma de Buenos Aires: Impresiones Buenos Aires.

National Geographic. (2004). La tierra de noche [Mapa]. 1:46.360.000. Washington, D.C.: National Geographic Maps.

National Geographic. (2005). África la huella humana [Mapa]. 1:14.636.000. Washington, D.C.: National Geographic Maps.

National Oceanic and Atmospheric Administration.(2019). Versión 1 VIIRS Day / Night Band Nighttime Lights. Recuperado de https://www.ngdc.noaa.gov/eog/viirs/download_dnb_composites.html

Nel.lo, O., López Redondo, J., Martín Oriol, J., \& Checa Rius, J. (2016). La luz de la ciudad. El proceso de urbanización en España a partir de las imágenes nocturnas de la Tierra. Barcelona: Universitat Autónoma de Barcelona.

Nel·lo, O., López, J., Martín, J., \& Checa,J.(2017). Energy and urban form. The growth of European cities on the basis ofnight-time brightness. Land Use Policy, 61, 103-112. doi:10.1016/j.landusepol.2016.11.007

Pérez Machado, R. P., Vizeu Barrozo, L., Luchiari, A., \& Small, C. (2014). Identification of the urban continuum of Sao Paulo Metropolitan Agglomeration with people, lights and land use. First results of a methodological approach. Annual Conference of the IGU Urban Commission (pp. 1-42). Poznan: IGU. Recuperado el 15 de Junio de 2019, de https://www.researchgate.net/publication/266202035 Identification_of_the_urban_continuum_of_Sao_Paulo_Metropolitan_Agglomeration_with_people_ lights_and_land_use_First_results_of_a_methodological_approach

Potere, D., Schneider, A., Angel, S., \& Civco, D. L. (2009). Mapping urban areas on a global scale: which of the eight maps now available is more accurate? International Journal of Remote Sensing, 30, 65316558. doi:10.1080/01431160903121134

QGIS Development Team. (1 de Noviembre de 2019). QGIS. Un Sistema de Información Geográfica libre y de Código Abierto. Recuperado de https://qgis.org/es/site/

Quaíni, K. (2014). Procedimiento metodológico para la generación de mapas de interfase urbanovegetación rural. Córdoba: Proyecto BPR - BID.

Roy Chowdhury, P. K., Maithani, S., \& Dadhwal, V. K. (2012). Estimation of urban population in IndoGangetic Plains using night-time OLS data. International Journal of Remote Sensing, 33, 2498-2515. doi:10.1 $\underline{080 / 01431161.2011 .616550}$

Sánchez de Miguel, A., Zamorano, J., Gómez Castaño, J., \& Pascual, S. (2014). Evolution of the energy consumed by street lighting in Spain estimated with DMSP-OLS data. Journal of Quantitative Spectroscopy and Radiative Transfer, 139, 109-117. doi:10.1016/j.jqsrt.2013.11.017 
Small, C., Elvidge, C. D., \& Baugh, K. (2013). Mapping urban structure and spatial connectivity with VIIRS and OLS night light imagery. Urban Remote Sensing Joint Event, (pp. 230-233). Sao Paulo. doi:10.1109/ JURSE.2013.6550707

Small, C., Pozzi, F., \& Elvidge, C. D. (2005). Spatial analysis of global urban extent from DMSP-OLS night lights. 96, 277-291. doi:10.1016/j.rse.2005.02.002

Sutton, P. C., Elvidge, C. D., \& Ghosh, T. (2007). Estimation of Gross Domestic Product at Sub-National Scales Using Nighttime Satellite Imagery. International Journal of Ecological Economics E Statistics, 8(S07), 5-21. Recuperado el 16 de Junio de 2019, de https://www.researchgate.net/publication/242254394_ Estimation_of_Gross_Domestic_Product_at_Sub-National_Scales_Using_Nighttime_Satellite_Imagery

Townsend, A. C., \& Bruce, D. A. (2010). The use of night-time lights satellite imagery as a measure of Australia's regional electricity consumption and population distribution. International Journal of Remote Sensing, 31(16), 4459-4480. doi:10.1080/01431160903261005 gov/

United States Geological Survey. (2019). Earth Explorer. Recuperado de https://earthexplorer.usgs.

Xin, X., Liu, B., Di, K., Zhu, Z., Zhao, Z., Liu, J., . . . Zhang, G. (2017). Monitoring urban expansion using time series of night-time light data: a case study in Wuhan, China. International Journal of Remote Sensing, 38(21), 6110-6128. doi:10.1080/01431161.2017.1312623

Zhou, Y., Smith, S. J., Zhao, K., Imhoff, M., Thomson, A., Bond-Lamberty, B., ... Elvidge, C. D. (2015). A global map of urban extent from nightlights. 10(5), 1-11. doi:10.1088/1748-9326/10/5/054011 